\title{
Experimental Study of Lamb Waves Propagation inside an Impact Damage in the Size of the Used Wavelength
}

\author{
S. Taleb $^{a, *}$, L. Rittmeier ${ }^{b}$, M. Sinapius ${ }^{b, c}$, F. Boubenider ${ }^{a}$, and D. Schmidt ${ }^{c}$ \\ ${ }^{a}$ Laboratory of Material Physics, Faculty of Physics, USTHB, Algeria \\ ${ }^{b}$ Institute of Adaptronics and Function Integration (IAF), TU Braunschweig, Germany \\ ${ }^{c}$ German Aerospace center (DLR e. V.), Braunschweig, Germany \\ *e-mail: sataleb@usthb.dz
}

Received August 21, 2019; revised October 16, 2019; accepted October 18, 2019

\begin{abstract}
The present work is an experimental study of Lamb waves propagation in an aluminum plate with an impact damage in the size of the S0 mode wavelength. The aim of this study is to visualize the wavefield near the defect in the case of extreme diffusion, as well as the interference of the modes inside of it and their transformation. The results were obtained by applying the continuous wavelet transform (CWT) on the wavefield data recorded by two ultrasonic scanning techniques: the air coupled ultrasonic (ACU) and the Laser doppler vibrometry (LDV), to obtain a C-scan and a B-scan of the plate respectively. Space-wavenumber representations showed the behavior of Lamb waves in the plate as well as the reduction in thickness of the impacted area. The width of the latter could be estimated and the modes present at each position of the plate could be identified.
\end{abstract}

Keywords: Lamb waves, interference, space-wavenumber, air coupled, laser

DOI: $10.1134 / \mathrm{S} 1061830920020084$

\section{INTRODUCTION}

Guided waves are widely used in non-destructive evaluation (NDE) techniques, Lamb wave [1-3] in particular, are used for the inspection of regions of constant thickness, any change of it causes variations in the phase velocity and the wavenumber. These variations signify that an abnormality is present in the structure, therefore, an analysis should be conducted to determine the severity of the defect to prevent any further harmful consequences.

Ultrasonic wavefield imaging has emerged in the last decade as a new NDE method, particularly, in the understanding of how ultrasonic guided waves propagate and interact with defects and structural features $[4,5]$. Multiple ultrasonic scanning techniques has been applied for the wavefield recording, we can mention, although the list is not inclusive: shearography [6], electronic pulse interferometry (ESPI) [7], air-coupled ultrasonic (ACU) [8, 9] and laser Doppler vibrometry (LDV) [10].

In the other hand, despite the fact that these techniques provide us with a visualization of the guided waves interaction with the damage in the form of $\mathrm{C}$-scans, the problem of the damage quantification imposes itself. For this reason, a variety of signal processing algorithms have been developed in the sake of a better understanding of the phenomena of wave-damage interaction, this includes, not for the exception, the reflection and mode separation method [11], standing wave analysis [12], wavenumber filtering [13-15] and local wavenumber filtering [16]. Although research activities in the structural health monitoring (SHM) have been conducted on impact damages $[17,18]$, they were more intensive on their effect on the physical properties of composite plates $[4,5,7,19]$, the specimen under test in this study is chosen to be an aluminum plate with a non-perforating impact damage. This choice was made under the motivation of studying the Lamb waves behavior in a configuration of extreme diffusion using guided waves [20], in order to obtain an interference phenomena of the wave caused by the internal multiple reflections of the diffused wave, depending on the relation between the used wavelength with the width and the remaining thickness in the bottom of the non-penetrating damage, which is certainly a topic of interest to the NDT/SHM community.

The aim of this study is to apply a continuous wavelet transform on the spatial data obtained from two different ultrasonic scanning techniques: ACU and LDV, in order to visualize the interference phenomena in such damage, and to have an estimation of its width and curvature, to localize it in the structure and to 


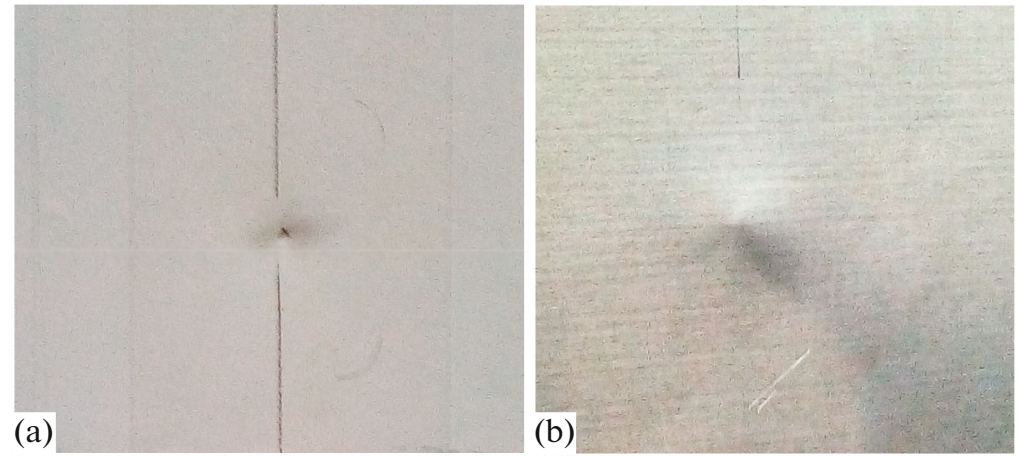

(c)

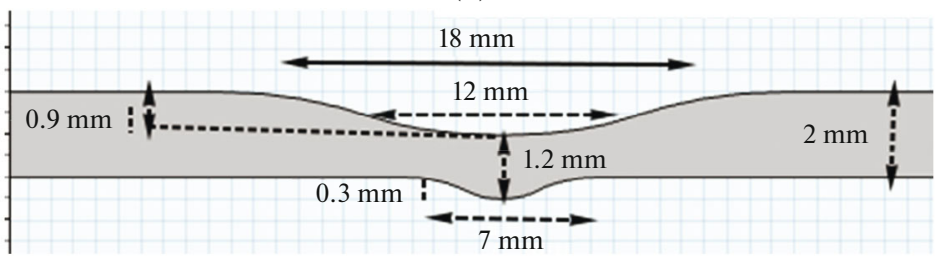

Fig. 1. Geometry of the waveguide: (a) Top view, (b) back view, (c) Vertical cut view.

identify the modes present at each position on the plate. In this paper, information about the ACU and LDV scanning systems are provided first. Next, wavenumber filtering using the CWT is presented, and the results of the analysis are discussed. These are followed by concluding remarks about the results obtained from the Space-wavenumber analysis of the wavefield data.

\section{EXPERIMENTAL SETUP}

\subsection{Geometry of the Waveguide and Lamb Waves Excitation}

The impact damage was introduced by a hemispherical projectile with an $8 \mathrm{~mm}$ radius. The projectile was shut by a pressure gun with energy of $4.4 \mathrm{~J}$. This value was chosen to be relatively weak to avoid perforation in the plate, but caused the bending of the plate outwards (Fig. 1b).

The width of the introduced impact damage was chosen to be close to the excited S0 mode wavelength which is about $12 \mathrm{~mm}$.

The studied waveguide is a squared aluminum plate of $1 \mathrm{~m}$ length and $2 \mathrm{~mm}$ thickness. The impact damage is introduced in the middle of the plate and its dimensions are represented in Fig. 1c.

The zero order symmetrical (S0) and anti-symmetrical (A0) modes were generated in the plate using a piezoceramic actuator. The actuator was excited with a 5 cycle tone burst in the LDV measurements, whereas in the ACU measurements, we have used a 25 cycle tone burst, both excitations were centered on $400 \mathrm{kHz}$. This excitation frequency corresponds to the non-dispersive range of the generated S0 mode shown in Fig. 2.

\subsection{Air Coupled Ultrasonic Measurements}

Air coupled ultrasonic technique (ACU) is a non-contact scanning method based on the sensing of dilatational waves emitted by the vibrating structure and detected by an ultrasonic microphone, however, the major challenge in using the ACU is the big difference in the acoustic impedance which increases the attenuation of the signal propagated from the plate to the microphone, the increase in the frequency used for the sensing of Lamb waves causes more attenuation in air [21].

To avoid this problem, the excitation frequency should be below $1 \mathrm{MHz}$. The system used in this experiment is the USPC 4000 Air Tech [8] as shown in Fig. 3a. We have attached a piezo-ceramic actuator to the back side of the plate to generate Lamb waves at $400 \mathrm{kHz}$ (Fig. 3b), while the wavefield was recorded on the front side of the plate, using the microphone for the sensing of the dilatational waves. The received signal that has been significantly weekend by the strong attenuation was amplified up to $50.2 \mathrm{~V}$ peak to peak, we have also taken care not to approach the edges of the plate to avoid reflections from the borders during the scan which took $6 \mathrm{~h}$. 


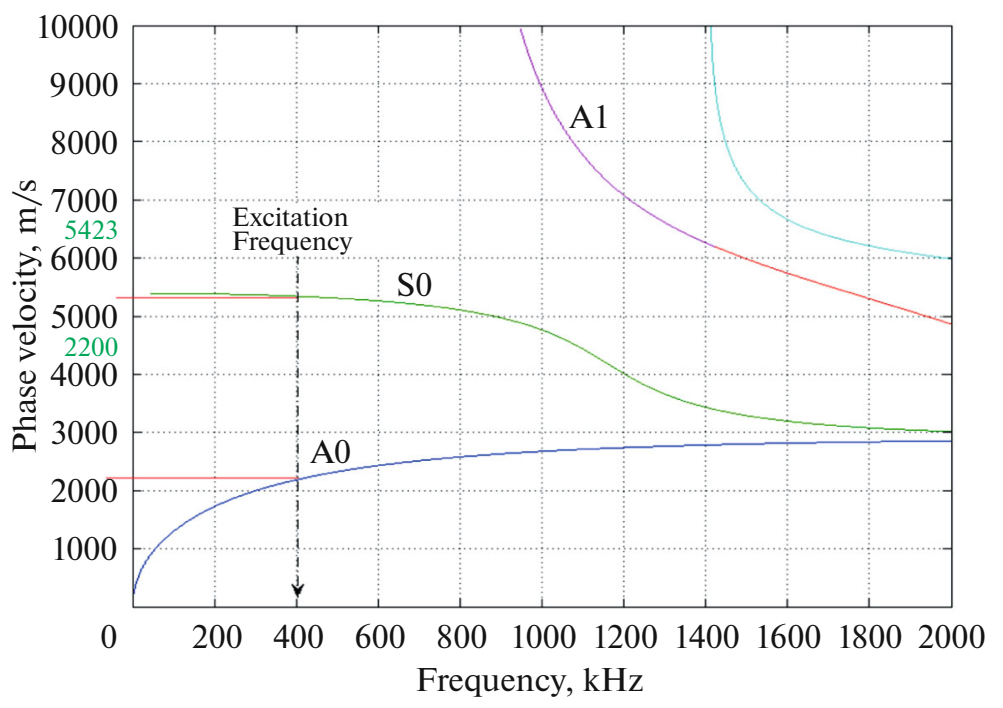

Fig. 2. Lamb waves dispersion curves.

(a)

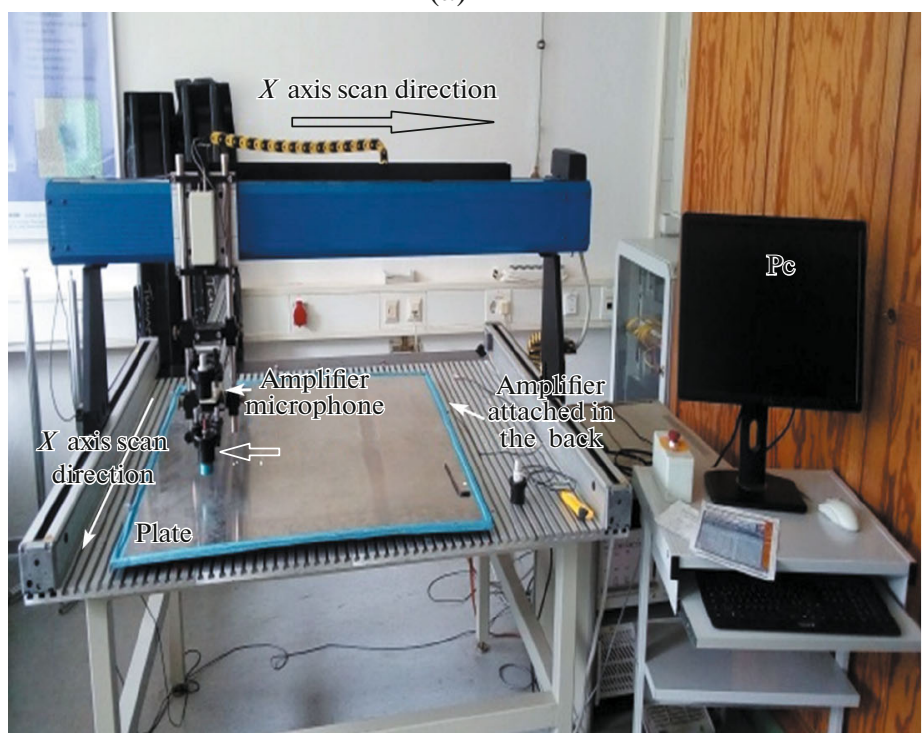

(b)

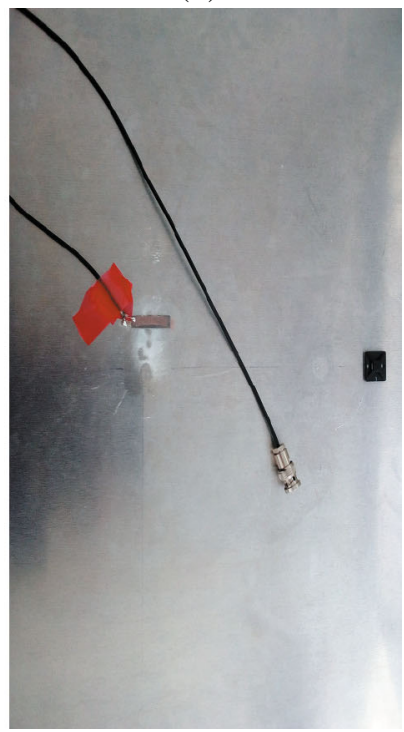

Fig. 3. (a) ACU experimental setup, (b) the actuator attached to plate.

This test was performed twice, one before introducing the damage, as a reference case, and the other after impacting the plate with the projectile, the measurement was undertaken with a time step of $0.105 \mathrm{~s}$ and at discrete positions of the P-wave resulting from the leaky Lamb wave, the data was recorded and processed in the USPC computer system which permitted the visualization of the propagation film, we have then taken snapshots from the film to obtain a C-scan of the area where the impact is present.

\subsection{Laser Doppler Vibrometry Measurements}

Unlike the ACU, the LDV is an optical system. It uses a laser beam to measure the out of plane component at different positions along the scanned area, the laser head is put in a direction perpendicular to the plate. We mention here that the used system for the measurement is Polytech PSV-500 (Fig. 4a) that measures displacements and velocities using single point data. For this experiment the LDV was used to measure displacements at discrete positions. 


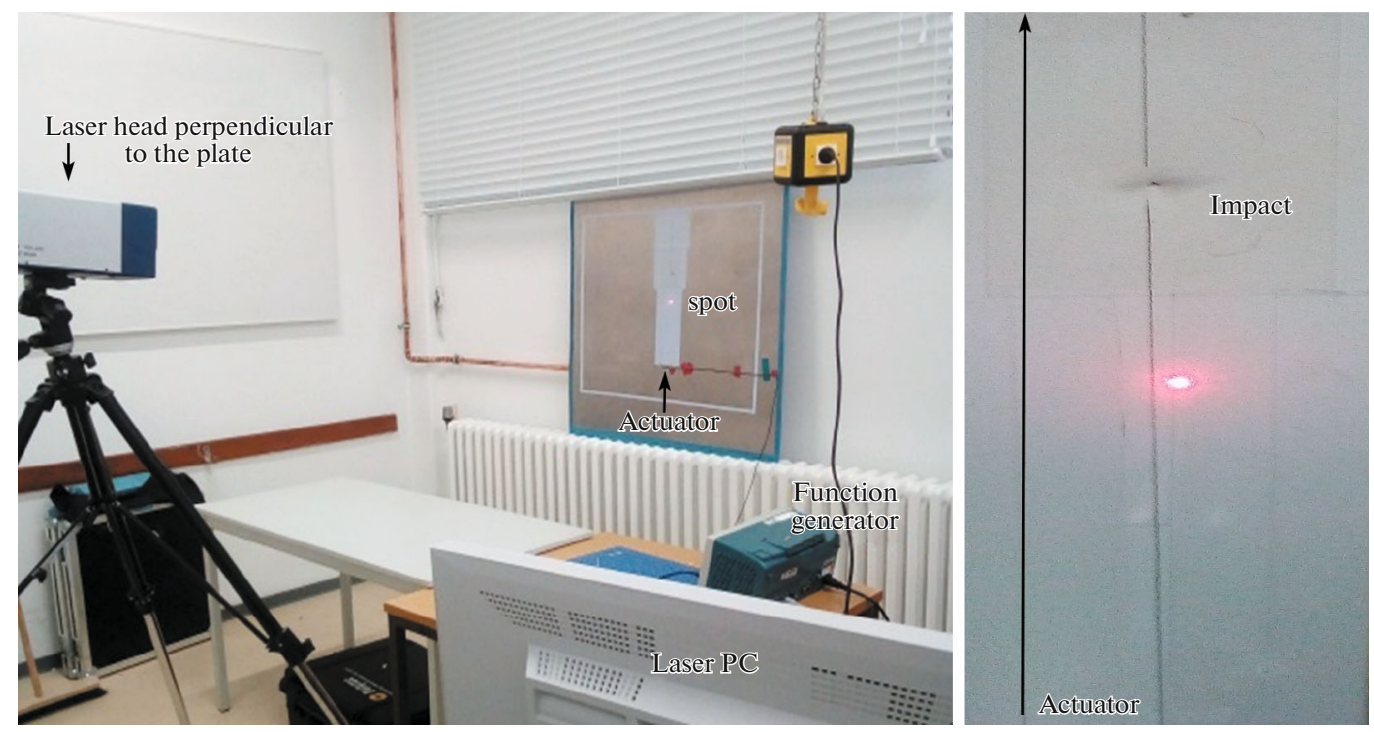

Fig. 4. (a) LDV experimental setup, (b) the scanned line for the LDV.

At each position, a separate signal was recorded, the time step taken for two successive measurements was $0.16 \mu \mathrm{s}$ and the spatial step was $0.58 \mathrm{~mm}$ for a $30 \mathrm{~cm}$ scanned segment, from the actuator throughout the impact as shown in Fig. 4b, which makes in total 513 discrete positions for the sensing, the total time taken for the full scan was $6 \mathrm{~h}$.

Each measured point gives a 1D data, we have constructed a 2D matrix using the separate $1 \mathrm{D}$ data obtained from the 513 measurements by concatenating them vertically, each column represents the obtained time signal at one discrete position, and each line of the same column represents the measured amplitude at a giving instant, Which results in a "Time-Position" matrix that contains the measured amplitude of the signal at each instant of the different discrete positions, and therefore a B-scan can be plotted.

\subsection{Wavenumber Filtering Method}

The analysis of time or space signals can be performed using the continuous wavelet transform (CWT) [13, 22]. The advantage of this function over the Fourier transform resides in its localization in time and space as it exists for a finite duration, centered on a chosen frequency allowing its stretching along the signal [23], and giving it the ability to determine any abrupt changes in it. This ability is especially interesting when we have spatial data, since any change in the geometry can be detected and localized in space. The formula of the one dimensional CWT with the coefficients $a$ and $T$ of a signal $s(t)$ is defined as:

$$
C(a, T)=\frac{1}{\sqrt{a}} \int_{-\infty}^{+\infty} s(t) \varphi^{*}\left[\frac{r-T}{a}\right] d t,
$$

where $a$ : scale, $T$ : translational value (any instant in time), $(t)$ : the wavelet function (the mother wavelet), the latter belongs to a big family of wavelets [24], each one of them has its own specific usage [25], in our case we have chosen the Morlet wavelet because of its two major advantages compared to other methods. First, the Morlet wavelet is Gaussian shaped in frequency domain which implies the absence of sharp edges that produce ripple effect and can be misinterpreted as oscillations; second, it is more computationally efficient [26]. The Morlet wavelet is defined as follows:

$$
\varphi(t)=\frac{1}{\sqrt{2 \pi}} e^{\frac{-t^{2}}{2}} e^{i 5 t}
$$

where the signal $s(t)$ is the 2D time-position matrix, the number of the measured points in the positions axes represents the scale a, which means that we have stretched the wavelet to fit the entire signal, and that is for the purpose of detecting the small values of the wavenumber, the transitional value $\mathrm{T}$ is referred to as $t_{1}$ and $t_{2}$ later in the following section, the steps taken in the measurements were used to rescale the pseudo wavenumber values calculated by the CWT to the real values of the wavenumber. 


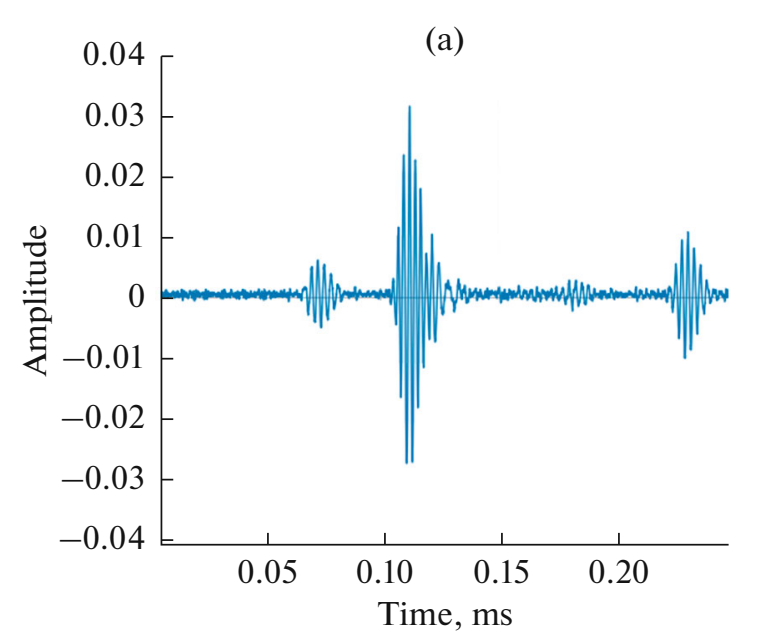

(c)

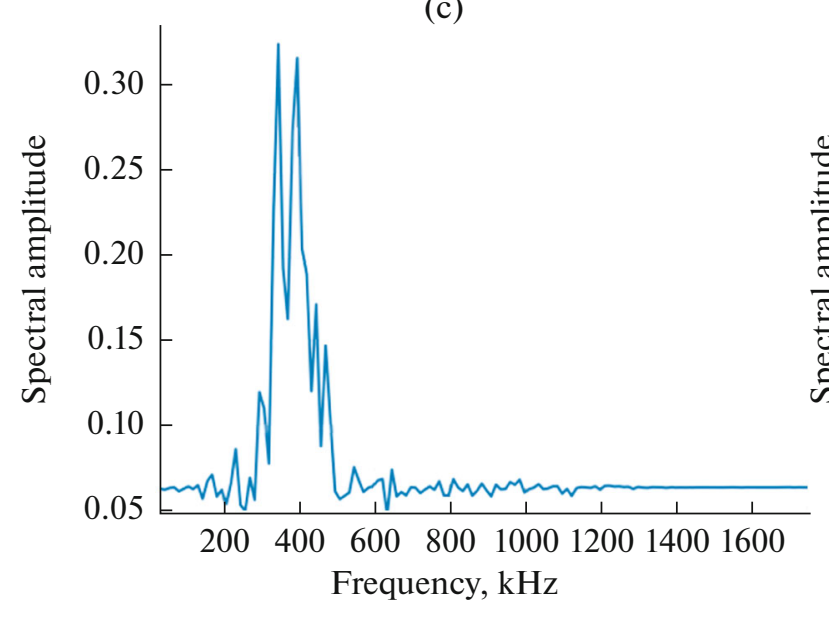

(a)

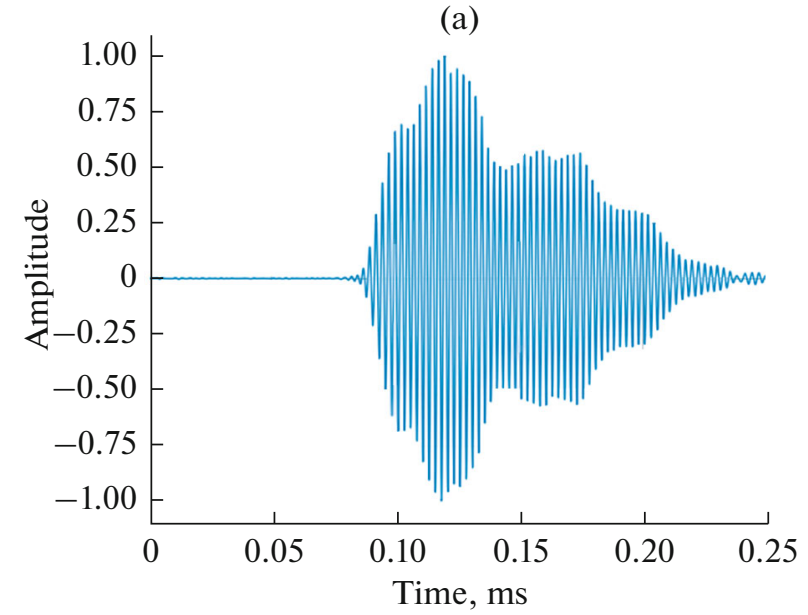

(d)

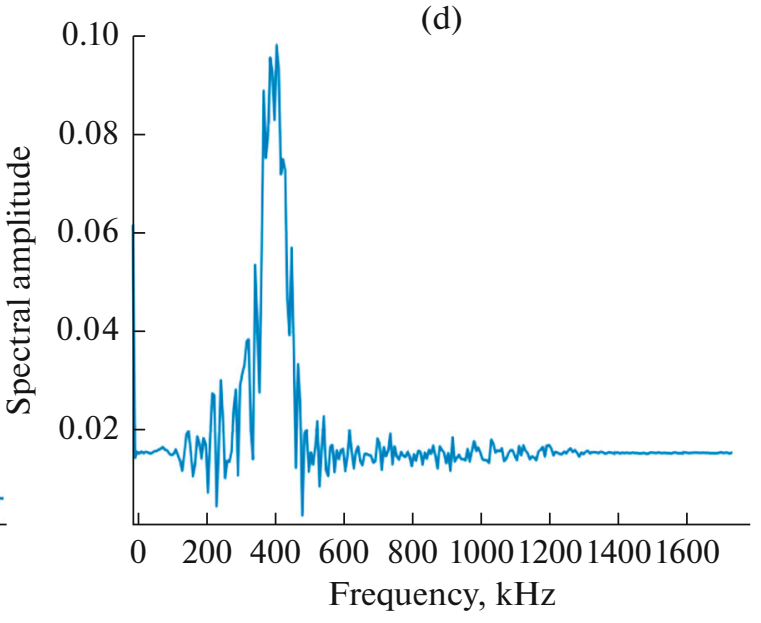

Fig. 5. (a) and (b) Signak received at a position post impact from the LDV and ACU respectively, (c) and (d) FFT of the LDV and ACU signals respectively.

To create a spectrogram, the wavelet maps the observations of the signal to a linear expansion of the coefficients, which are related to pseudo frequencies and time. The magnitude of these coefficients is proportional to the energy of the signal at a certain time [13]. The same principle is applied when the signal gives spatial quantities; at every position the CWT calculates the corresponding wavenumber, which results in a space-wavenumber representation [27, 28]. We mention that all scripts for the data analysis were written under Python 3 using the information provided in the Sections 2.2 and 2.3.

\section{RESULTS AND DISCUSSION}

The time signal obtained from the LDV at a position post-defect is represented in Fig. 5a. The S0 mode wave packet appears prior to the $\mathrm{A} 0$ one, because it is the fastest propagating mode, whereas in Fig. $5 \mathrm{~b}$, the wavepackets are more intense and it is difficult to clearly distinguish between the two modes. A simple FFT of the time signals in Figs. $5 \mathrm{c}$ and $5 \mathrm{~d}$ shows that it is well centered on the excitation frequency of $400 \mathrm{kHz}$ in both measurements.

We have then used the LDV to acquire a B-scan of the damaged plate (Fig. 6), as we have explained before in Section 2.3, this figure shows the propagation of the ripples in the plate and throughout the defect region, which is defined by the $Z$ form resulting from the multiple reflections of the wavepackets inside of it.

The zero order Lamb modes, are known to be adiabatic modes [28], which signifies that they exhibit the same behavior locally in sections with varying thickness as in a waveguide with a constant thickness, allowing their propagation inside regions with variable thicknesses and their possible trapping in cases of extreme diffusion. In this case, we can distinguish between the propagation of two modes; the fastest coming ripples with respect to time are denoted as S0 wapackets, whereas the A0 wavepackets are the slower 


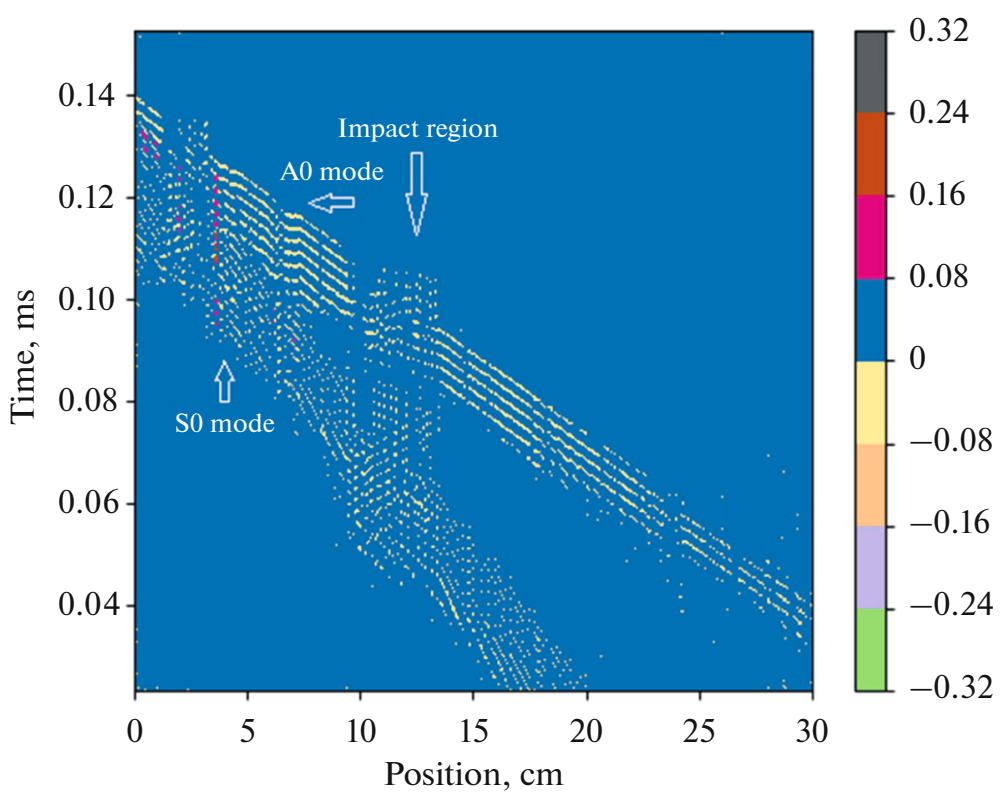

Fig. 6. B-scan results from LDV.

ones. The defect is detected by both modes as the latter ones get reflected multiple times inside of it, which shows that the interaction of the Lamb modes with such configuration is possible. Yet, the identification of the resulting transmitted modes for both wavepackets in the area post defect cannot be clearly seen from this figure, and will be discussed later after applying the CWT on the data.

The ACU results are shown as snapshots of the wavefield after post processing. Figure 7a shows a scan of the plate before introducing the impact damage which serves as a reference case. The $\mathrm{S} 0$ mode propagates faster than the A0 mode; both modes have circular wavefronts as a result of the isotropy of the material.

The ACU measures leaky P-wave displacements which makes it more sensitive to the A0 mode displacements than the S0 ones, this results in a better contrast of the A0 mode wavefronts compared to the $\mathrm{S} 0$ mode. Whereas in Fig. 7b which represents a C-scan of the damaged plate, the behavior of the S0 and A0 modes changes at the position $(x, y)=(450,550) \mathrm{mm}$. At this point, the scattering from the impact is visible as a result of the energy trapping and the reflections from the edges of the impact [4], the wavefield at the impact is composed from the incident $\mathrm{S} 0$ and $\mathrm{A} 0$ modes and the reflections at the edges. Although the impact was detectable and visible from the scan shown in Fig. 7b, information about the form of the impact and the resulting modes from the interaction are still needed.

In order to understand what happened at the edge and inside the impact region, and to be able to identify the modes present at every scanned position of the plate, a CWT was applied on the B-scan obtained from the LDV measurements as well as on the data extracted from the ACU C-scan, and that is for the same chosen segment shown in Fig. 4b, in order to compare between both results at two different instants $t_{1}$ and $t_{2}$ of the propagation time which are defined as: $t_{1}=1.69 \mathrm{~ms}$ and $t_{2}=2.15 \mathrm{~ms}$ for the ACU measurements, and, $t_{1}=2.4 \mathrm{~ms}$ and $t_{2}=2.72 \mathrm{~ms}$ for the LDV measurements. We mention that the time $t_{1}$ refers to the instant when the wavepackets reach the impact region, as for $t_{2}$, the wavepackets got transmitted from it. Sketches of the plate above the figures are not to scale.

Figure 8 a represents the space-wavenumber plot at $t_{1}$, it gives the value of the wavenumber at each position of a $45 \mathrm{~cm}$ scanned line With this analysis, we can identify the modes present in the plate at the regions before, inside, and after the impact, the wavepackets at the region before the impact, which varies from 0 to $23 \mathrm{~cm}$, are centered on the wavenumber values equal to 470 and $1150 \mathrm{~m}^{-1}$. These two values of the wavenumber correspond to the theoretical wavenumber values of the S0 and A0 modes respectively which are calculated as follows:

$$
k=\frac{2 \pi f}{V_{\varnothing}},
$$

where $f$ is the excitation frequency, and $v$ is the phase velocity, which is shown in Fig. 3. This confirms that the two excited modes are indeed the S0 and A0. 
(a)

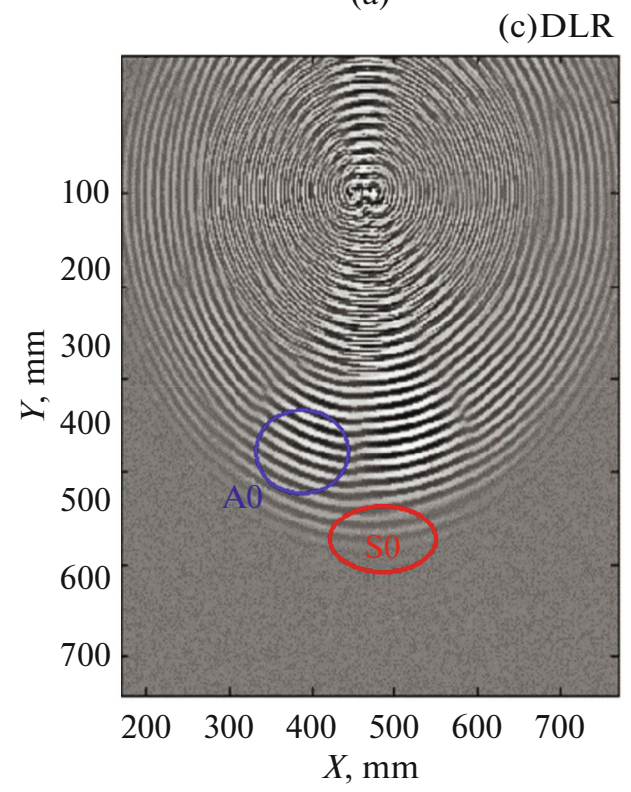

(b)

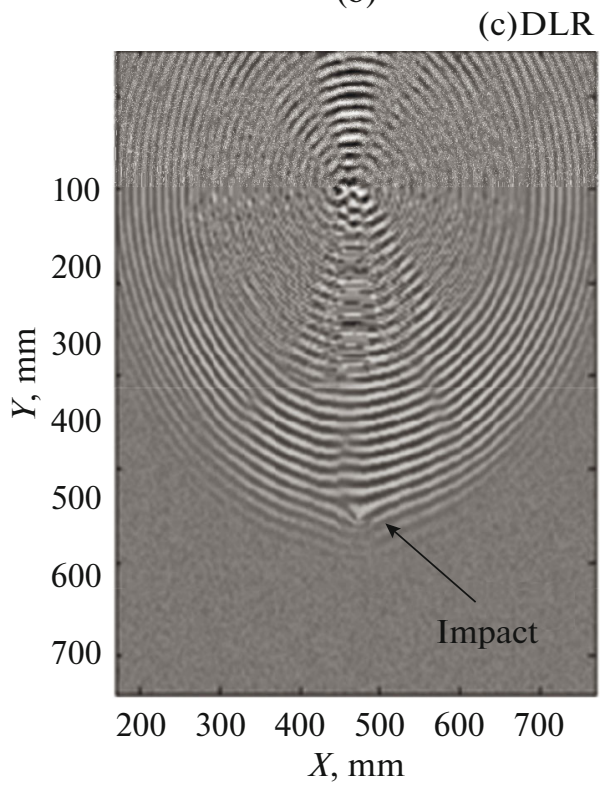

Fig. 7. C-scans of (a) undamaged plate, (b) damaged plate.

(a)

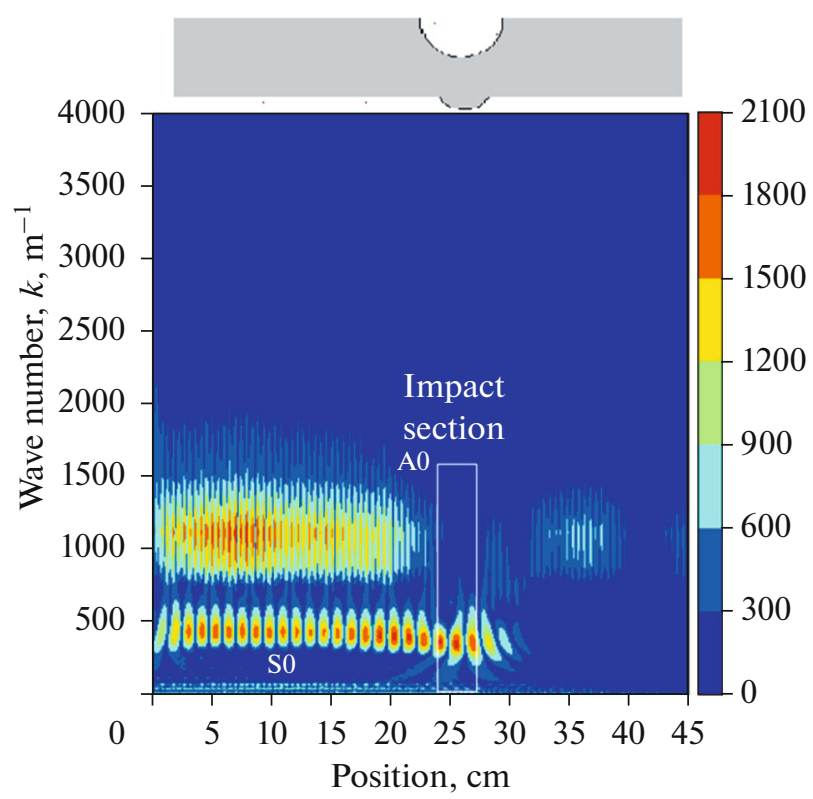

(b)

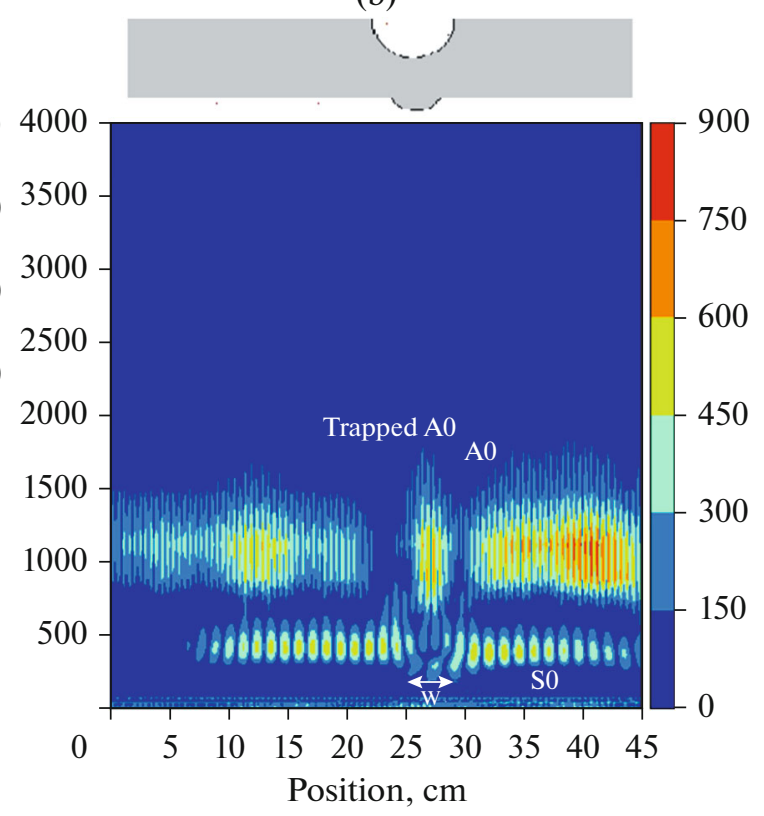

Fig. 8. Wavenumber filtering of the ACU results at (a): $t_{1}$, (b) $t_{2}$.

The S0 mode wavepackets propagate along the plate and get distorted as they reach the impact region, that presents a reduction in thickness, and undertake its geometrical form. At this position, equal to $28.5 \mathrm{~cm}$ new wavepackets emerge from the $\mathrm{S} 0$ ones and take the value of the $\mathrm{A} 0$ mode wavenumber, which signifies that a part of the S0 mode has been converted into an A0 mode as a result of the asymmetrical shape of the impact. By the incidence section, at the position $23 \mathrm{~cm}$ and contrary to the $\mathrm{S} 0$ mode, the A0 mode wavepackets do not seem to have undertaken the form of the thickness reduction of the impact. We can intuitively explain this by the main difference in the way the compressional S0 mode and the A0 flection mode propagate when the waveguide is no longer symmetric. 
(a)

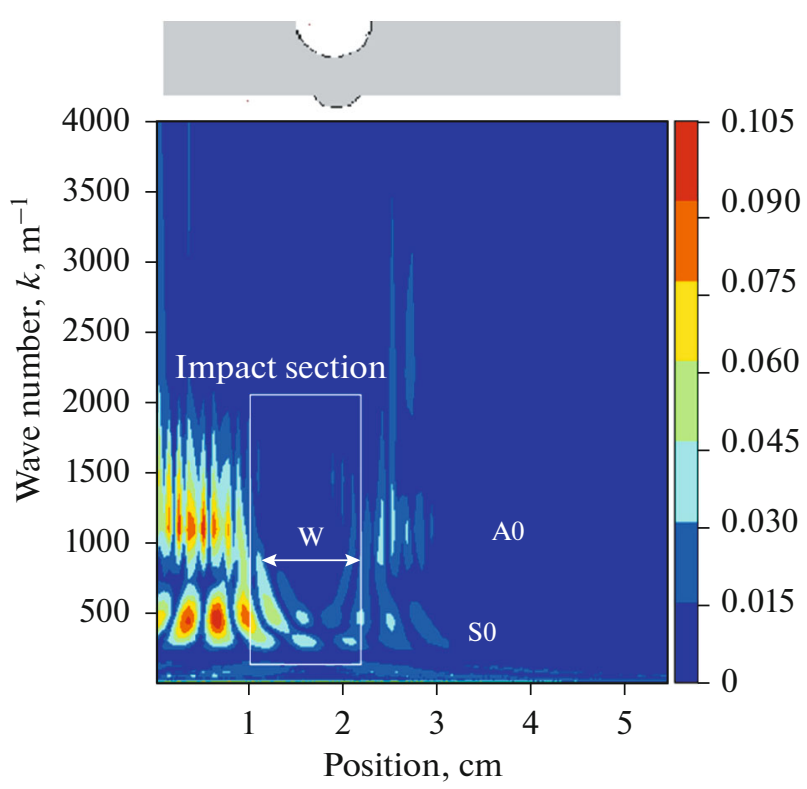

(b)

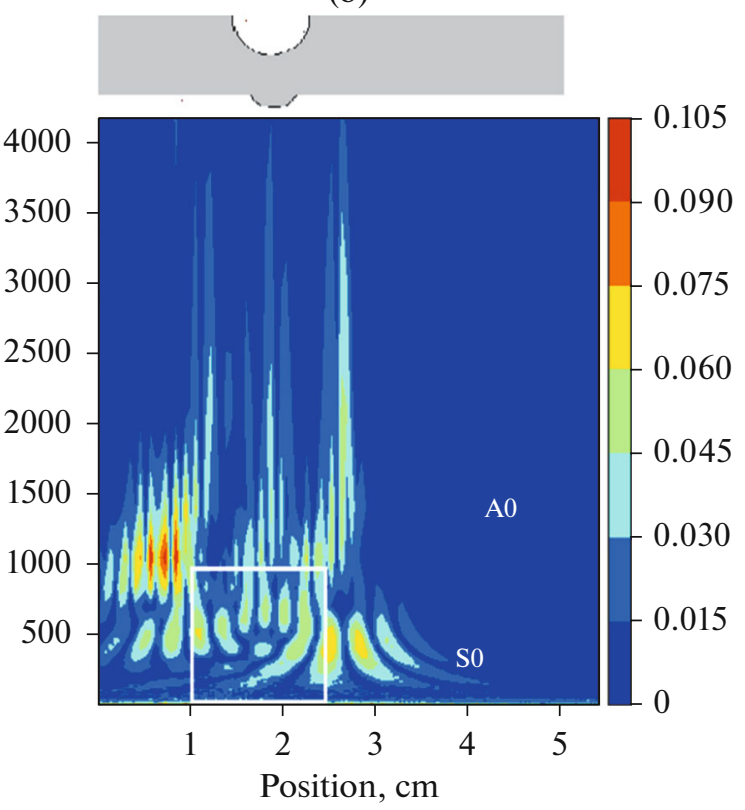

Fig. 9. Wavenumber filtering of the impacted area from the LDV results at (a) $t_{1}$, (b) $t_{2}$.

At the time $t_{2}$ represented in Fig. $8 \mathrm{~b}$. The results shown in this figure reinforce the previous findings. The A0 mode doesn't reshape the curvature of the impact unlike the S0 mode that continues propagating inside the impact section, and loses a part of its energy to the new emerging anti symmetrical displacements of the A0 mode. The interference of the S0 and A0 wavepackets is observed from this figure as the wavefield near the defect is strongly distorted, and the assignation of their corresponding wavenumber is rather difficult, both modes get transmitted afterwards out of the impact region. The geometry of the impact is given by the form of the $\mathrm{S} 0$ wavepackets. The width of the impact can therefore be estimated by calculating the difference in position between the two edges of the curvature, which is denoted in the figures as "W".

Similarly to the analysis done on the ACU measurements, the LDV measurements are shown in Fig. 9, where the impact section was zoomed to a $5.5 \mathrm{~cm}$ scaled line of the region varying from position $=23-$ $28.5 \mathrm{~cm}$, to have a better estimation on the width of the impact, in addition, the authors did not have enough wavefield data from the LDV measurements to fully compare them with the ACU ones, due to the available capacity of the used LDV equipment.

The $\mathrm{A} 0$ and $\mathrm{S} 0$ modes were identified at the incidence section, the S0 mode propagates through the impact region, whose width is estimated here to be $w=12 \mathrm{~mm}$, which is approximately the width of the impact in Fig. 1c at the efficient section of the impact.

In Fig. 9b, at the first edge of the impact, the two modes interfere with each other as a result of the sudden change in the plate's thickness, the S0 undertake the curvilinear form of the impact, therefore, its width and location can be estimated as we have mentioned before, the A0 mode wavepackets get distorted inside the impact section as they emerge from the S0 ones. The conversion of the $\mathrm{S} 0$ mode into an $\mathrm{A} 0$ mode is a result of the relocation of the S0 mode energy in the form of anti-symmetrical displacements in an attempt to escape its trapping inside the impact region, giving both symmetrical and symmetrical displacements.

Results from both set of measurements were compatible, the interference of the modes was observed from the space-wavenumber analysis done on the recorded wavefield data, yet, the ACU results were better in terms of clarity, also, the distinction between the two used modes was better visualized here as the wavepackets did not get distorted as in the LDV measurements.

\section{CONCLUSIONS}

This paper investigates the behavior of Lamb waves in a plate presenting a non-perforating impact damage in the size of its wavelength, the results were obtained by applying a space-wavenumber analysis based on the continuous wavelet transform of the wavefield data recorded by the air coupled ultrasonic and the laser Doppler vibrometry scanning techniques. 
The interference of Lamb wave modes was visualized inside the impact region, which represents a configuration of extreme diffusion, and the propagating modes were identified along the plate. We were also able to estimate the width of the impact, as well as the reduction in thickness and the curvature from the behavior of the $\mathrm{S} 0$ wavepackets, shown in the space-wavenumber representations.

\section{ACKNOWLEDGMENTS}

This paper is a result of the collaboration between the IAF of TU Braunschweig, Germany and LOA laboratory, USTHB, Algeria.

We would also like to thank the German Aerospace center (DLR e. V.) for helping with the projectile impact and the air-coupled experiments.

\section{REFERENCES}

1. Viktorov, I., Rayleigh and Lamb Waves, New York: Springer US, 1967.

2. Rose, J.L., Ultrasonic Waves in Solid Media, Cambridge: Cambridge Univ. Press, 1999.

3. Auld, B., Acoustic Fields and Waves in Solids, Melbourne: FL: Robert E. Krieger Publ. Comp., 1990.

4. Michaels, J., Ultrasonic wavefield imaging: Research tool or emerging nde method? [Electronic Resource], in AIP Conf. Proc., 2017, vol. 1806, no. 1, pp. 020001-1-020001-14. URL: https://aip.scitation.org/doi/pdf/ 10.1063/1.4974542? class=pdf. Accessed February 16, 2017.

5. Ren, Y., Qiu, L., Yuan, S., and Bao, Q., On-line multi-damage scanning spatial-wavenumber filter based imaging method for aircraft composite structure, Materials, 2017, vol. 10, no. 5, pp. 519-540.

6. Focke, O., Hilderbrand, A., and Kopylow, C., Inspection of laser generated lamb waves using sheriographic interferometry [Electronic Resource], in 1st Int. Symp. Laser Ultrasonics: Sci. Tech. Appl., Montreal, July 16-18, 2008. URL: http://citeseerx.ist.psu.edu/viewdoc/download?doi=10.1.1.157.4301\&rep=rep1\&type=pdf

7. Lammering, R., Observation of piezoelectric induced lamb wave propagation in thin plates by use of speckle interferometry, Exp. Mech., 2010, vol. 50, no. 3, pp. 377-387.

8. Hillger, W., Buhling, L., and Ilse, D., Air-coupled ultrasonic testing method, system and practical applications [Electronic Resource], in 11th Eur. Conf. Nondestr. Test., Prague, October 6-10, 2014. URL: https://www.ndt.net/events/ECNDT2014/app/content/Paper/482_Hillger.pdf

9. Szewieczek, A., Heinze, C., and Sinapius, M., Guided wave displacement validation for shm application using air coupled scanning technique, in Int. Workshop Struct. Health. Monit., Stanford, September 1-3, 2015.

10. Kang, T., Moon, S., Han, S., Jeon, J., and Park, G., Measurement of shallow defects in metal plates using interdigital transducer-based laser-scanning vibrometer, $N D T \& E$ Int., 2018, vol. 102, pp. 26-34.

11. Asakawa, E., Mizohata, S., Tsujimoto, S., Mikada, H., and Nishizawa, A., Depth imaging of obs reflection data with wave field separation, in 12th Int. Symp. Recent Adv. Explor. Geophys., Kyoto, January 17, 2008.

12. Jeon, J., Jung, H., Kim, D., Park, G., Kang, T., and Han, S., Experimental study on guided and standing waves based full field laser scanning for damage detection [Electronic Resource], in 9th Eur. Workshop Struct. Health. Monit., Manchester, July 10-13, 2018. URL: https://www.ndt.net/article/ewshm2018/papers/0322-Jeon.pdf

13. Rogge, M. and Johanston, P., Wavenumber imaging for damage detection and measurement [Electronic Resource], in AIP. Conf. Proc., Burlington, July 17-22, 2011, vol. 1430, no.1, pp. 761-768. URL: https://pdfs. semanticscholar.org/61e0/78aa79a8490f6028556362713d2935cba817.pdf

14. Tian, Z., Yu, L., Lamb wave frequency-wavenumber analysis and decomposition, J. Intell. Mater. Syst. Struct., 2014, vol. 25, no. 9, pp. 1107-1123.

15. Michaels, T., Michaels, J., and Ruzzene, M., Frequency-wavenumber domain analysis of guided wavefields, Ultrasonics, 2011, vol. 51, no. 4, pp. 452-466.

16. Fan, G., Zhang, H., Zhu, W., and Chai, X., Lamb wave local wavenumber approach for characterizing flat bottom defects in a isotropic thin plate, Appl. Sci., 2018, vol. 8, no. 9, pp. 1600-1626.

17. Senyurek, V., Detection of cuts and impact damage at the aircraft wing slat by using lamb wave method, Measurement, 2015, vol. 67, no. 5, pp. 10-23.

18. Monnier, T., Lamb waves-based impact damage monitoring of a stiffened aircraft panel using piezoelectric transducers, J. Intell. Mater. Syst. Struct., 2008, vol. 17, no. 5, pp. 411-421.

19. Diamanti, K., Hodgkinson, J., and Soutis, C, Detection of low-velocity impact damage in composite plates using lamb waves, Struct. Health Monit., 2004, vol. 3, no. 1, pp. 33-41.

20. Xeridat, O., Etude expérimental de la propagation, de la diffusion et de la localisation des ondes de lamb (Experimental study of the propagation, diffusion and localization of Lamb waves). Dissertations and Theses, Nice : Nice Sophia Antipolis Univ., December 2011. URL: https://tel.archives-ouvertes.fr/tel-00652619/document 
21. Hillger, W., Ilse, D., and Buhling, L., Practical applications of air-coupled ultrasonic technique [Electronic Resource], in 4th Int. Symp. NDT Aerospace, Augsburg, November 13-14, 2012. URL: https://www.ultrasonic.de/ article/aero2012/papers/p11.pdf

22. Pramila, T., Shukla, R., Kishore, N., and Raghuram, V., A study of the spectral behavior of laser-generated Lamb waves using wavelet transforms [Electronic Resource], in 4th Int. Conf. NDT, Chania, October 11-14, 2007. URL: https://www.ndt.net/article/hsndt2007/files/Pramila.pdf

23. Rhif, M., Ben Abbes, A., Farah, I., Martinez, B., and Sang, Y., Wavelet transform application for/in non-stationary time-series analysis: a review, Appl. Sci., 2019, vol. 9, no. 7, pp. 1345-1366.

24. Chui, C., Wavelets: A Tutorial in Theory and Applications, Texas: Academic Press, 1992.

25. Dogra, A., Goyal, A., and Agrawal, S., Performance comparison of different wavelet families based on bone vessel fusion, Asian J. Pharm., 2016, vol. 10, no. 4.

26. Cohen, M.X., A better way to define and describe morlet wavelets for time-frequency analysis, Neuroimage, 2019, vol. 199, pp. 81-86.

27. Marical, P., Ech-Cherif El-Kettani, M., and Predoi, M., Guided waves in elastic plates with gaussian section variation: experimental and numerical results, Ultrasonics, 2007, vol. 4, nos. 1-4, pp. 1-9.

28. Hamitouche, Z., Ech-Cherif El-Kettani, M., Izbicki, J., and Djelouah, H., Reflection at the Cut-off and Transmission by tunnel effect in a waveguide with linear section variation, Acta Acust. Acust., 2009, vol. 95, no. 5, pp. 789-794. 